\title{
Understanding as an uncertainty overcome
}

\section{A. D. Kiyatkina, V. D. Shadrikov ${ }^{1}$}

${ }^{1}$ HSE University, 20 Myasnitskaya Str., Moscow 101000, Russian Federation

DOI: $10.18255 / 1996-5648-2021-3-434-443$

Research article

Full text in Russian

The article discusses understanding as a human tendency to remove uncertainty through the phenomenon of «entropy». The learning process initially puts the student in a situation of constant movement from a disordered environment to an ordered one, which occurs due to the constant interruption of the student's inner world balance. Studies of understanding through entropy allow us to consider the subject of understanding as an irreversible dynamic system, then the individuality of pedagogical influence can be explained by the degree of stability of this system. Taking into account the variety of individual differences that determine the process of understanding, the idea of building a probabilistic model of understanding is proposed.

Keywords: understanding; uncertainty; entropy; dynamical systems

\section{INFORMATION ABOUT AUTHORS}

\author{
Kiyatkina, A. D. | Postgraduate \\ Shadrikov, Vladimir D. | E-mail: shadrikov@hse.ru \\ Full member of the RAO, Doc. Sc. (Psychology), Professor
}

Funding: RFBR, project 20-313-90043. 


\title{
Понимание как преодоление неопределённости
}

\section{А. Д. Кияткина ${ }^{1}$, В. Д. Шадриков ${ }^{1}$}

${ }^{1}$ Национальный исследовательский университет «Высшая школа экономики», ул. Мясницкая, д. 20, Москва, 101000, Российская Федерация

DOI: 10.18255/1996-5648-2021-3-434-443

УдК 159.9

Научная статья

Полный текст на русском языке

В статье рассматривается понимание как стремление к снятию неопределенности через френомен «энтропии». Процесс обучения изначально ставит ученика в ситуацию постоянного движения от неупорядоченной среды к упорядоченной, что происходит за счет постоянного нарушения равновесия во внутреннем мире ученика. Исследования понимания через энтропию позволяют рассматривать субъекта понимания как необратимую динамическую систему, тогда индивидуальность педагогического воздействия может объясняться степенью устойчивости этой системы. С учетом многообразия индивидуальных различий, детерминирующих процесс понимания, предлагается идея о построении вероятностной модели понимания.

Ключевые слова: понимание; неопределенность; энтропия; динамические системы

ИНФОРМАЦИЯ ОБ АВТОРАХ

\author{
Кияткина, А. Д. | Аспирант \\ Шадриков, Владимир Дмитриевич $\mid$ E-mail: shadrikov@hse.ru \\ Действительный член РАО, доктор \\ психологических наук, профессор
}

Финансирование: РФФИ, проект № 20-313-90043.

Классическая наука подчёркивала порядок и устойчивость. Законы формулировались, исходя из принципа детерминированности событий во Вселенной. В настоящее время вера в определённость ставится под сомнение, подчёркивается фондаментальная роль вероятности. Достаточно (c) Кияткина А. Д., Шадриков В. Д., 2021

Статья открытого доступа под лицензией CC BY (https://creativecommons.org/licenses/by/4.0/) 
Кияткина А. Д., Шадриков В. Д.

подробно эту тенденцию описывает Илья Пригожин. «Человечество достигло поворотного пункта, - пишет он, - начало новой рациональности, в которой наука более не отождествляется с определённостью, а вероятность - с незнанием» [1, с. 13]. В результате перехода на вероятностное описание предмета изучения мы «получаем более приемлемое описание, в котором есть место и для законов природы, и для новаций и творческой активности» [1, с. 21].

Преодоление неопределённости связано с понятием энтропии. Энтропию, пишет Марри Гелл-Манн, «можно рассматривать как своего рода меру незнания» [2].

В образовательном процессе ученик постоянно двигается от неупорядоченной среды (незнания) к упорядоченной (знанию), разрешая задачи, которые ставятся перед ним учителем. И каждая новая задача возвращает его к неупорядоченному состоянию, идёт постоянное нарушение равновесного состояния и перевод ученика в неравновесное состояние, в состояние частичного незнания. Это неравномерное состояние ученик разрешает, приобретая опыт решения задач определённого класса.

Специфика неравновесных состояний состоит в том, что они характерны только для ученика, а не для учителя.

Важным является положение об устойчивом и неустойчивом движении. «Если не вдаваться в детали, - пишет И. Пригожин, - то можно сказать, что устойчивыми динамическими системами называют такие, у которых небольшие изменения начальных условий порождают небольшие эфрфекты. Но у обширного класса динамических систем небольшие возмущения начальных условий со временем усиливаются [1, с. 32]. Каждый отдельный ученик является такой системой. Одно и то же педагогическое воздействие у различных учеников может дать (и дает) совершенно различный эффект. В отдельных случаях этот эффект носит отставленный и длительный характер. В этом заключается индивидуальная реакция на педагогическое воздействие. В настоящее время этот эфрфект недостаточно осознан и изучен. Но важно знать о его существовании. Важно также знать, как этот эффект проявляется в условиях группового обучения. Другим следствием данного положения является вывод о том, что педагогические (и психологические) закономерности должны носить статистический характер: описание индивидуального поведения и учебной группы будут носить различный характер.

В описываемой ситуации важно отнести того или иного ученика к определённому типу реагирования на стандартное педагогическое воздействие - к реагированию по типу устойчивой или неустойчивой динамической системы. 
Понимание как преодоление неопределённости...

«Почему для того, чтобы прийти к обобщению законов природы, включающему в себя необратимость и вероятность, потребовалось так много времени? - спрашивает И. Пригожин. Одна из причин носит идеологический характер - желание достичь в нашем описании природы квазибожественной точки зрения» [1, с. 38]. Другая причина заключалась в отсутствии адекватного математического аппарата в области функционального анализа.

В психологии и педагогике это проявилось в стремлении перейти к методу эксперимента по типу естественных наук, что сопровождалось резким снижением глубины проникновения в психологическую сущность поведения, уходом от наиболее сложных проблем психологии.

Важным методологическим фрактором является положение, заключающееся в том, что, чтобы описать индивидуальную траекторию поведения, необходимо точно описать начальные условия. Но именно этого мы и не можем сделать (по крайней мере, сегодня). Поэтому мы и должны признать необходимость вероятностного описания последствий педагогических воздействий.

Математики, исследующие динамические системы, показали, что динамические системы в большинстве своем не интегрируемы. Главную роль в интеграции играет существование резонансов между степеняли свободъ [3]. Но «неинтегрируемость прокладывает путь к новой, статистической фрормулировке законов динамики» [1, с. 41].

Если мы теперь будем рассматривать наш мозг как динамическую систему, то можно поставить вопрос о том, что выступает интегратором в его деятельности при решении определённых задач. Можно предположить, что мозг как система в целом не интегрируется, а в каждой задаче объединяются статистические компоненты, причем у каждого индивида по-своему. А механизмом интеграции выступают мотивация и переживания. Это во многом проясняет механизм сознания. Неинтегрируемость открывает новый путь к объяснению динамики сознания. Повышая уровень мотивации и переживаний, мы увеличиваем пространство мыслей, вовлекаемых в решение проблемы.

Современная психология слабо отражает реальность, когда дело касается сложных систем, таких как личность, поведение, сознание и т. п. Это скорее построения авторов конкретных моделей, отражающих отдельные стороны реальности, но не поведение человека в различных ситуациях.

Концепции динамики личности исходят из того, что человек представлен как обратимая динамическая система, практически не изменяющаяся со временем. Но это не так, человек представляет собой необратимую динамическую систему, для которой характерна «стрела времени». В качестве компенсации предложены методы лонгитюда и поперечных срезов. 
Но это грубая характеристика динамики развития. Выход из данной ситуации находится на пути внедрения вероятностных идей для описания поведения человека.

«Устойчивые системы, порождающие определённость, соответствуют только идеализациям, или аппроксимациям .... Никакая фрормулировка законов природы, не учитывающая конструктивную роль времени, не может считаться удовлетворённой» [1, с. 53-54].

Исследования в области поведения динамических систем показали, что на некотором расстоянии от равновесия система утрачивает устойчивость. «Точка, где происходит потеря устойчивости, называется точкой бифуркации. За точкой бифуркации начинается множество новых явлений», образование новых пространственно-временных образований, которые И. Пригожин назвал диссипативными структурами [1, с. 63].

Такие точки бифуркации наблюдаются и в развитии человека. Следствием бифуркаций может появиться новое решение. Эти точки выступают как точки креативности. Неустойчивое состояние субъекта, состояние поиска приемлемого решения, может порождать новые мысли и новые решения. Особенно это относится к выбору новых видов поведения.

«Бифуркации - это проявление внутренней дифференциации между частями самой системы и системы и окружающей её средой» $[1$, c. 66$]$. Такая бифуркация может наблюдаться внутри функциональной системы нервной деятельности, образования новых связей в системе головного мозга, порождающих новые решения. Это следует из высказанного ранее положения об отсутствии всеобщей связи между элементами (нейронами и нейронными ансамблями) головного мозга. Важно подчеркнуть, что здесь мы имеем выход на механизм, объясняющий креативность. «Бифуркации можно считать источником диверсификации и инновации» [4]. В явлениях бифуркации заложен механизм самоорганизации. Именно на этой основе созданы превосходные механизмы адаптации живых существ и человека. «Природа действительно связана с созданием непредсказуемых новаций, где возможное богаче реального» [1, с. 68].

Важную роль в описании развития играет поток корреляций (динамика корреляций). С таким потоком корреляции мы сталкиваемся, когда изучаем развитие деятельности. Эта динамика корреляций отражает развитие деятельности со стороны внутренних условий [5]. Динамика корреляций отражает и статистическое описание развития.

Важную роль в педагогическом процессе играют процессы диссеминаций, когда в процессе общения и совместной деятельности психические качества одного участника отражаются (влияют) на изменении таких же качеств другого (других) участника совместной деятельности. В частности, в образовательном процессе мышление учителя влияет на мышле- 
ние учеников и мышление каждого ученика влияет на мышление других (и мышление класса учеников в целом). $\mathrm{K}$ сожалению, эти явления остаются практически неизученными. Можно предположить, что креативность является не только качеством индивидуальности, но и качеством коллективного субъекта деятельности.

Необратимость в протекании динамических процессов приводит к эбббектам длителъной памяти. Это наглядно наблюдается в учебной деятельности. Но следует помнить, что при общей направленности на реализацию программы в процессе обучения всегда можно вернуться к изученному материалу за счет повторения, но при этом следует учитывать, какие изменения к этому времени произошли в ученике.

Описывая развитие науки как диалога человека с природой, И. Пригожин пишет: «Классический идеал науки - мир без времени, памяти и истории - напоминает тоталитарные кошмары, описанные Олдосом Хаксли, Миланом Кундерой и Джорджем Оруэллом» [1, с. 135]. Это полностью относится и к психологии, ведущим разделом которой должна стать психология развития. И нам следует гораздо больше внимания уделять не бытию, а становлению.

Рассмотрим теперь процесс понимания с другой стороны - со стороны определённости - неопределённости ситуации, в которой находится человек. В своей повседневной жизни человек стремится к определённости. Неопределённость порождает чувство тревоги, страха, которое отражается в определённых эмоционалъны состояниях. Для изучения понимания в контексте педагогической ситуации мера определённости - неопределённости учебного материала является особо актуальным параметром [6].

Как отмечает Т. В. Корнилова, в настоящее время интенсивно развивается «новая область психологического знания - психология неопределённости» [7, с. 8]. В рамках данного направления «было поставлено много новых вопросов: каким образом риск проявляется в стратегиях принятия решений?; способствует ли он продуктивным решениям человека?; рационально ли поступает человек, идущий на риск?; связан ли поведенческий риск с бездумными действиями человека или с его необдуманным решением идти на риск?; как поступают более «умные» люди в ситуации рискованного выбора?; о каком уме в первую очередь нужно здесь говорить - о его житейском понимании, представленном в имплицитных теориях, или об изменениях, которые дают интеллектуальные тесты?; в какой степени в регуляцию мыслительной деятельности в условиях неопределённости и риска включаются мотивы и эмоции человека? [7, с. 8].

Большинство этих исследований проведено на модели принятия решения в условиях выбора в ситуации неопределённости. Как отмечает Т. В. Корнилова, «человек в условиях неопределённости не может 
Кияткина А. Д., Шадриков В. Д.

следовать сложившимся в индивидуальном опыте клише действий, выборов или решений; психологическая регуляция его активности необходимо включает в такой ситуации (как бы широко она ни понималась) предвосхищение, прогнозирование неизвестного, выдвижение гипотез и цели, а значит, новообразования, посредством которых совершается актуалгенезис выбора. Области решения проблем и принятия решений предполагают разные аспекты проявления и изучения интеллектуально-личностного потенциала человека. С последней областью связывается представление о неопределённости оснований выбора, а не только множества возможных исходов [7, с. 9].

Нам представляется важным предложенное Т. В. Корниловой деление на решение проблем и принятие решений (ПР). В каждом из этих направлений проведено значительное количество исследований, опираясь на которые можно констатировать, что между выделенными проблемами существуют не только различия, но и много общего, так как решение проблем также предполагает принятие определённых решений.

В исследовании понимания на первое место выходит решение проблемъ понимания. И рассматривается понимание как процесс достижения понимания в условиях неопределённости. Но понимание это не выбор, а раскрытие сущности, процесс же понимания выступает как снятие неопределённости, в которой скрыта сущность. Необходимо согласиться с утверждением Т. В. Корниловой, что при этом проявляются разные аспекты интеллектуально-личностного потенциала человека.

Исходя из того, что есть понимание, мы можем перейти к систематическому рассмотрению детерминант, обусловливающих процесс и результат понимания. Отталкиваясь от идеи о том, что понимание представляет собой разновидность деятельности, мы можем утверждать, что одной из главных детерминант является резулътат деятелъности. Результат вначале представляет как цель. Отсюда следует, что для понимания важно задание цели деятельности ученика. И эта цель, в свою очередь, должна быть понята. Следовательно, в достижении понимания существенную роль играет цель, способ её задания и понимание цели. Таким образом, иель деятельности является следующей детерминантой процесса понимания.

Важной детерминантой понимания является определение началънъх условий в деятельности и характеристик субъекта. Это связано с выяснением «зоны ближайшего развития» [8], когда учитель знает, как решать учебную задачу и что для этого необходимо, но этого не знает ученик. Именно с подготовкой ученика к усвоению нового содержания на основе актуализации данных, необходимых для решения задачи, и начинается освоение нового материала. Это начинается с выяснения того, каким ресурсом рас- 
полагает ученик по отношению к цели урока. Важно знать, как ученик может пользоваться своим ресурсом. Второй важный аспект выяснения начальных условий - установление способов включения нового материала в содержание внутреннего мира. Этот этап также определяется ресурсом ученика, прежде всего содержанием его психики. Роль учителя заключается в помощи ученику установить связи старого и нового материала (подчеркнём, что мы не занимаемся проблемой решения учебной задачи, мы занимаемся проблемой понимания старого и нового).

K начальным условиям относится также мотивация деятельности и личностные качества, способствующие пониманию.

В проблеме мотивации важно учитывать, что ученик обладает природной мотивацией $к$ снятию неопределённости.

К личностным качествам следует отнести прежде всего когнитивные способности, способности преодоления стресса (стрессоустойчивость), рефлексивность, волевые качества, навыки решения учебных задач. Из когнитивных способностей следует выделить память и мышление. Память включает в себя содержание психики, она играет особую роль в организации всей психической деятельности [9]. Память определяет содержание психики.

Мышление определяет процессуальную сторону понимания. Именно в процессах мышления достигается связь старого и нового. В совместной работе мыиления, паляти и переживаний достигается понимание.

Для эмпирического исследования процесса понимания и его детерминант первоочередной задачей является выделение признаков понимания и соотнесение их с результатами понимания [10]. Тесно связанным с процессом понимания является определение понимания как способности. Способность к пониманию можно рассматривать как метаспособность, интегрирующую ряд познавательных способностей, как вид одарённости, обеспечивающей понимание (одарённость обычно связывают с конкретной деятельностью, в данном случае одарённость связана с универсальным видом деятельности - пониманием).

При изучении процесса понимания важно выяснить отношения между знаниями и пониманием и отношения процессов решения задачи (РЗ) принятия решения (ПР) и понимания учебного материала (ПУМ).

Знание - есть результат познавательной деятельности, заключающийся в выделении свойств предметов объективной реальности, и на этой основе выделения самих вещей в окружающем мире, установление законов и закономерностей происхождения и развития объектов объективного мира, включающих самого познающего, выяснение возможностей их использования в своей жизнедеятельности. 
Кияткина А. Д., Шадриков В. Д.

Понимание - это установление личностного смысла приобретаемого знания, уяснение происхождения и возможностей его практического использования.

Сопоставляя процессы ПР и РЗ, мы должны отметить, что в первом случае субъект деятельности располагает определённым количеством альтернатив решения проблемы и ему необходимо сделать выбор между ними; во втором случае он должен сам отыскать путь решения проблемы. Несомненно, что различия между принятием решения и решением задачи относительное. В каждом ПР имеются элементы решения задачи, и наоборот. Но все же различать их необходимо.

Понимание также присутствует и в ПР и в РЗ, но имеет свою сущность. Понимание заключается в том, что оно направлено на осмысление того, что получено в результате ПР и РЗ, на поиск личностного смысла результата в обоих случаях.

Учитель, как правило, знает, какие процедуры и операции необходимо использовать при решении задачи. Но нужно, чтобы их нашел ученик, а учитель должен создать условия, которые помогут ученику найти эти процедуры и операции. В качестве такой помощи может выступить подсказка: как лучше разбить на части условия задачи, с какого пункта целесообразнее начать поиск решения (так как могут существовать причины, требующие приступить к решению именно с этого пункта), в какой последовательности по отношению к частям решать задачу, учитывая, что решение каждой частной задачи (подзадачи) расширяет информационную базу для решения других подзадач. Возможны случаи, когда следует приостановить решение частной задачи, которая решается с трудом, и вернуться к ней на более позднем этапе, после решения других подзадач. Здесь самое важное - использовать полученную информацию как оперативную базу для решения последующих задач. Если ученик не может решить задачу, учитель должен найти близкую ей, но более легкую.

Программу решения учебной задачи можно представить как цепь взаимосвязанных вероятностных суждений. Умение выстраивать такую цепь является не только одним из ведущих учебных умений, но и основой научной деятельности. Чем длиннее эта цепь, тем больше вероятность успеха найти нетривиальное решение. Примерами этого являются решения шахматных задач выдающимися гроссмейстерами.

Таким образом, переход на конструирование вероятностных моделей успешности понимания по принципу неустойчивых динамических систем может открыть новые возможности для его измерения. Понимание как преодоление неопределенности позволяет анализировать специфику понимания учеником нового материала, так как ученик в процессе обучения постоянно сталкивается с разрешением неопределенности. Бо- 
лее того, ученик приходит в класс с уже имеющимся индивидуальным опытом, который индивидуализирует понимание. Для изучения понимания через метафору динамической системы в педагогическом контексте важны ранее рассмотренные феномены: точка бифруркации (как точка индивидуального творчества) и диссеминация (отражение психических качеств одного человека в другом).

Раскрытие неопределенности через понятие энтропии открывает возможности количественного измерения ее влияния на успешность понимания учебного материала, что, в свою очередь, приближает нас к созданию инструментов обучения с целью понимания.

\section{Ссылки}

1. Пригожин И. Конец определённости. Время, хаос и новые законы природы. M.; Ижевск, 2000. 208 с.

2. Gell-Mann M. The Quark And The Jaguar. L.: Little, Brown, 1994. 392 p.

3. Пуанкаре А. Новые методы небесной механики // Избранные труды. М.: Наука, 1971. Т. 1.771c.

4. Николис Г., Пригожин И. Познание сложности. М.: Мир, 1990. 334 с.

5. Рубинштейн С. Л. Основы общей психологии. Москва: Питер, 1999. 720 с.

6. Кияткина А. Д. Неопределенность как мотивация учения и фрактор понимания учебного материала. Ярославская психологическая школа: история, современность, перспективы: сборник материалов Всероссийской научной конференции. Ярославль: Филигрань, 2020. С. 398-401.

7. Корнилова Т. Б. Интеллектуально-личностный потенциал человека в условиях неопределённости и риска. СПб.: Нестор-История, 2016. 344c.

8. Выготский Л. С. Педагогическая психология / под ред. В. В. Давыдова. М.: Педагогика, 1991.480 с.

9. Бехтерева Н. П. Здоровый и больной мозг человека. Л.: Наука, 1980. 260 с.

12. Макарова К. В. Психология понимания. М.: Правда-Пресс, 2020. 166 с. 